\title{
Color coated glazing for next generation BIPV: performance vs aesthetics
}

\author{
Benjamin Riedel ${ }^{1,2}$, Paul Messaoudi ${ }^{1}$, Ya Brigitte Assoa ${ }^{1}$, Philippe Thony ${ }^{1}$, Rayan Hammoud ${ }^{3}$, \\ Laure-Emmanuelle Perret-Aebi ${ }^{4}$, and John A. Tsanakas ${ }^{1, *}$ (D) \\ ${ }^{1}$ CEA-INES, Department of Solar Technologies, 50 avenue du Lac Léman, 73370 Le Bourget-du-Lac, France \\ 2 Université Savoie Mont-Blanc, LOCIE UMR CNRS 5271, 50 Avenue du Lac Léman, 73370 Le Bourget-du-Lac, France \\ 3 Saint-Gobain Sekurit, Glasstrasse 1, 52134 Herzogenrath, Germany \\ ${ }^{4}$ EPFL STI IMT PV-LAB, Rue de la Maladière 71B, 2002 Neuchâtel, Switzerland
}

Received: 5 July 2021 / Received in final form: 2 November 2021 / Accepted: 16 November 2021

\begin{abstract}
Through the H2020 BE-SMART project, we work on the validation and industrialization of new materials (and processes) for manufacturing next-generation cost-efficient, reliable and highly aesthetic/ performing BIPV. On this basis, we aim at introducing novel multifunctional and transformative BIPV elements, in the concept/form of Energy Positive Glazing (EPoG). The project's developments so far indicate the high potential of e.g. using colored encapsulants, interferential filter technique and/or ceramic-based colored glazing for implementing novel "transformative" BIPV with high aesthetic quality. Yet, since BIPV's primary function is electricity production, we need to understand and quantify the impact of such coloration solutions on the performance (and reliability, in longer terms) of future BIPV. In this paper, we present an experimental comparative study on the optical and electrical performance of multiple color coated and patterned BIPV glazing solutions, towards their upscaling and commercialization. In particular, we performed optical transmission measurements and light intensity-/angle-depent IV characterization on 25 different colored glass samples and 10 different colored/patterned glass PV laminates respectively. The measurement results and their discussion presented in this paper provide valuable insights into the optical-electrical performance of the investigated colored BIPV glazing, as well as a first identification of BIPV industry-relevant colors and patterns with the best potential "compromise" between aesthetics and performance, for future energy positive glazing applications.
\end{abstract}

Keywords: Building-integrated PV (BIPV) / optical characterization / electrical characterization / BIPV glazing

\section{Introduction - rationale and aim of the study}

BIPV industry has intensified R\&D efforts, the last few years, towards the technical maturity and commercialization of several off-the-shelf products for façade and roof integration. Yet, "breakthrough" developments are scarce, while market penetration and success have so far remained well below expectations, with even some remarkable casualties such as those of Dow's Powerhouse, BP's solar shingles or Scheuten Solar. With a closer look at the BIPV market and R\&D landscape, we identify certain barriers and setbacks of diverse origins, throughout the whole BIPV value chain, that eventually have been holding back the

\footnotetext{
* e-mail: ioannis.tsanakas@cea.fr
}

broader diffusion and uptake of BIPV. These barriers range from the design/development and product/installation level (e.g. complex product designs non-adapted for buildings, limited insights into reliability/performance, reluctance of architects), up to the end-use and policymaking level (lack of coordination in standardization and among stakeholders in the building value chain) $[1,2]$.

To address such challenges, a shift in mindset from PV to the building industry is an essential first step. With this in mind, through the EU-funded project BE-SMART we aim to introduce novel multifunctional and transformative BIPV, in the concept/form of Energy Positive Glazing (EPoG) [3]. The latter shall thus replace (or transform) traditional passive building materials (e.g. tiles, aluminum façade or standard architectural glass) by innovative active elements. Towards this concept, a central R\&D activity in BE-SMART is to validate and industrialize new materials 
and processes for manufacturing next-generation BIPV. Particular focus is drawn on implementing novel "transformative" EPoG approaches such as revolutionary white or colored BIPV glazing.

So far, BIPV have been presenting a very limited diversity in terms of visual appearance - their vast majority being either of black or dark blue color - which itself introduces a major constraint to the aesthetic (beyond the physical) integration of BIPV in the architectural concept of buildings. On this basis, it is very relevant (yet challenging) for BIPV aesthetics - being a subjective notion - to conceive a metric in order to best assess the preference of the greatest number of people, in terms of visual comfort or simply appeal. For the latter, apart from visual perception of different colors, textures or the light reflection, another important notion and influencing factor is the environment around the building [4]. In overall, the idea of aesthetic BIPV developments is to identify colors and textures that enable seamless "visual" integration of BIPV in the building's environment and architecture, e.g. by harmonizing with the surrounding color shades or by imitating traditional materials for buildings, such as wood or stone [5].

It might be tempting to simply introduce materials or designs for highly aesthetic BIPV e.g. in multiple colors or with customized, diverse visible features or textures. Yet, this is not a straightforward task, but a rather complex challenge, especially when BIPV performance (and reliability) comes into the equation, next to BIPV aesthetics. Even a seemingly small change to the color, to a visible feature or to a pattern/ texture of a PV component, affects the quantity and "quality" (i.e. spectral range) of the incident light absorbed/reflected by the PV module; which, in turn, can have a major impact on the PV module's generated current/power. Indeed, a part of the solar spectrum is reflected and/or absorbed before arriving on the cell, which involves a loss of efficiency compared to the theorical limit. In fact, this loss is colordependent, considering that each color reflects its own part of the incident light spectrum [5]. Thus, in the pursuit of an ideal BIPV "performance vs aesthetics" compromise, it is necessary to employ colors that correspond to a high efficiency and/or develop coloring procedures and materials that minimize the impact on the quantity of light transmitted to the solar cells, while also offering costefficiency and reliability. Today's landscape in colored BIPV developments and their R\&D challenges have been studied and reported recently by IEA PVPS Task 15 experts [6].

In this paper, we present an experimental comparative study on the: (i) optical performance, (ii) intensity-/angledependent electrical performance of multiple color coated and patterned BIPV glazing solutions, towards their upscaling and commercialization.

\section{Methodology overview/experimental set-up}

\subsection{Optical characterization study}

We performed measurements on 25 square colored glass samples, fabricated and provided by an industrial partner of BE-SMART, with identical dimensions (area) of $200 \times 200 \mathrm{~mm}^{2}$ and a thickness of $4 \mathrm{~mm}$. The colored
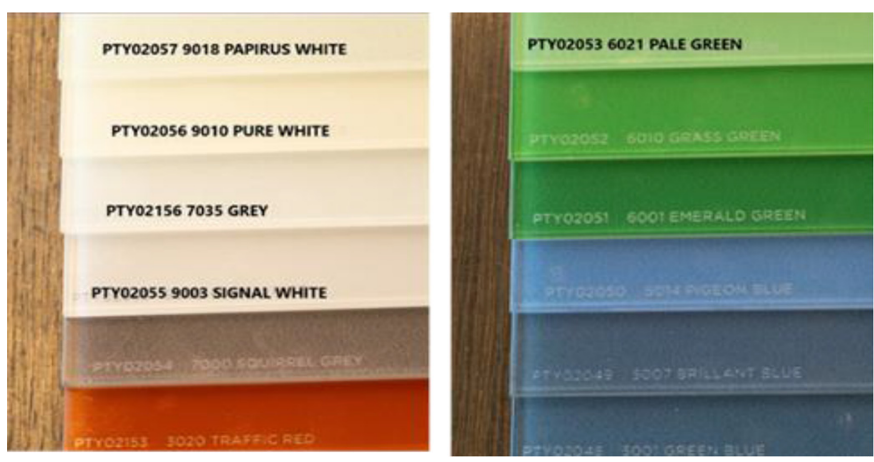

Fig. 1. The colored coating RAL of the measured glass samples.

coating of such glass is ceramic-based digital printed, fully covering one (front) side of the glass, using hightemperature fire enamel process. The studied RAL code variations are shown in Figure 1. We performed two different measurement sets:

- Set 1: on 12 samples, colored using the standard digital printing "recipe" of the industry partner.

- Set 2: on 12 samples, colored with new updated digital printing "recipe", with an additional 13th measurement was carried out on a glass sample coated with grey tint color.

For this study, we employed a PerkinElmer LAMBDATM $1050+$ UV/Vis/NIR spectrophotometer set-up, in order to measure, extract and compare the transmittance and reflectance spectra of the different RAL codes/paints. This set-up features an integrating sphere which allows to spatially integrate radiant flux in scatter transmission and diffuse reflectance sample measurements. As such, we can obtain indirect measurements of the transmission/reflectance spectra. For the latter, we use a calibration gauge (i.e. a reflection "standard"), placed behind the measured samples, in order to retrieve the measurements. We therefore need to determine the spectral characteristics of such standard, as well as those of the glass on which the paint is coated. In such setting, the analysis consists of two measurements, M1 and M2, through which we can determine the transmittance and the reflectance of each measured sample. An overview of the experimental setting for obtaining the M1 and M2 is given in Figure 2.

For the M1, there are three steps: (i) measurement of the reflectance of the standard; (ii) measurement of the reflectance of each colored glass sample, with the standard placed behind it; (iii) acquisition of the measurement M1. Besides, the measured M1 is physically expressed by the following equation:

$$
M 1=R_{v}+T_{v}^{2} \cdot R_{p}+T_{v}^{2} \cdot T_{p}^{2} R_{e}
$$

where $R_{v}$ and $T_{v}$ are respectively the reflectance and transmittance of the colored glass (which are both measured at this step, thus become known) and $R_{e}$ is the reflectance of the standard, also measured and thus known. Yet, in the above equation (which corresponds to the obtained measurement M1), we still have two unknown parameters, i.e. the $R_{p}$ and the $T_{p}$, which are actually the 


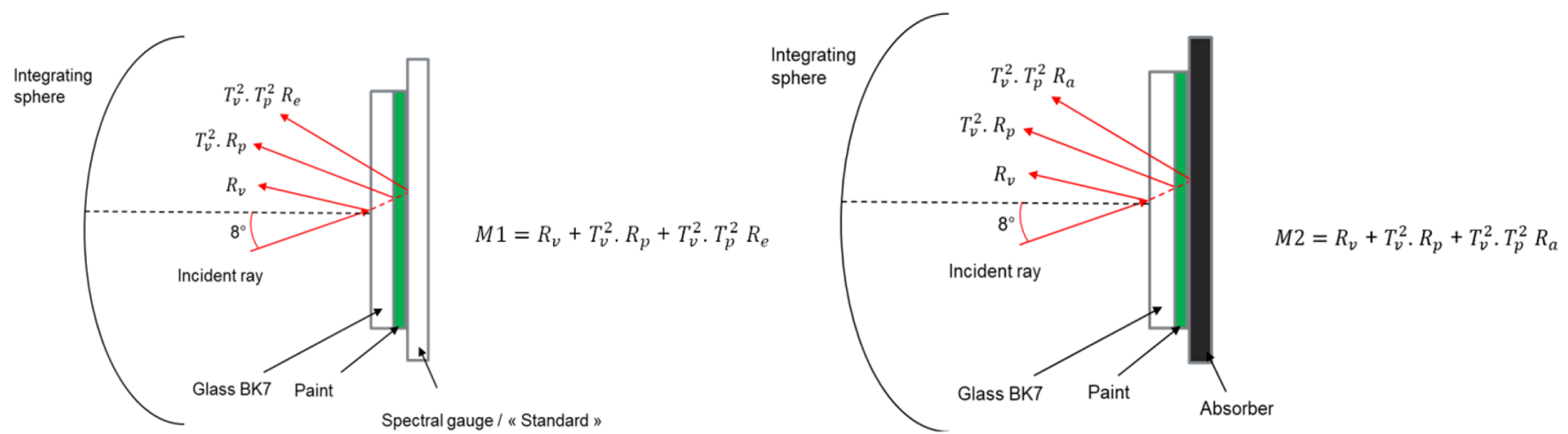

Fig. 2. Overview of the experimental setting for the measurements M1 (left) and M2 (right).

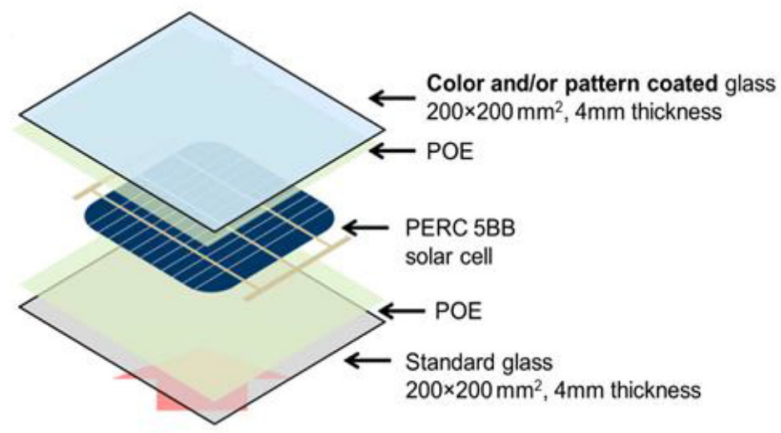

\begin{tabular}{cl}
\hline Module & Color code and/or pattern \\
\hline$\# 1$ & standard (uncoated) glass $\rightarrow$ "Reference module" \\
$\# 2$ & RAL9018 Uniform (100\% coverage) \\
$\# 3$ & RAL9018 Squared pattern \\
$\# 4$ & RAL9018 Dotted pattern \\
$\# 5$ & RAL9018 Linear pattern \\
$\# 6$ & RAL6010 Uniform (100\% coverage) \\
$\# 7$ & RAL6010 Dotted pattern \\
$\# 8$ & RAL7000 Uniform (100\% coverage) \\
$\# 9$ & RAL7000 Dotted pattern \\
$\# 10$ & tint (grey) glass \\
\hline
\end{tabular}

Fig. 3. The applied layout for the different PV laminates of the study (left) and the 10 colored/patterned glazings (right).

reflectance and the transmittance of the paint coating which are looking for, for the different RAL cases. We therefore look to extract a second measurement/equation. This leads us to the M2.

In the case of the M2, there are two steps: (i) measurement of each colored glass sample, with a standard (known) absorber body/film behind it; (ii) acquisition of the measurement M2. The measured M2 is physically expressed by the following equation:

$$
M 2=R_{v}+T_{v}^{2} \cdot R_{p}+T_{v}^{2} \cdot T_{p}^{2} R_{a}
$$

where $R_{v}$ and $T_{v}$ are respectively the measured/known reflectance and transmittance of the colored glass, while $R_{a}$ is the reflectance of the absorber, also measured and known at this step.

As, we end up having two equations (corresponding to the measurements M1 and M2), with the reflectance $R_{p}$ and the transmittance $T_{p}$, of the paint coating that we look for, as the two unknown parameters of this $2 \times 2$ system:

See equation (1) below.
Obviously, apart from the reflectance and the transmittance spectra of the paint coatings, we can also determine the absorbance $A_{p}$ (or emissivity) spectrum as well, as it is known that the sum $A_{\mathrm{p}}+R_{\mathrm{p}}+T_{\mathrm{p}}$ equals to 1 .

\subsection{Electrical characterization study}

For this experimental study, we fabricated 10 single-cell PV laminates, each differentiated by 9 distinct colored and/or patterned coatings on their front glass, along with 1 individual "reference" PV laminate sample of with standard (uncoated) glass. The chosen layout/BOM for the fabrication of the PV laminates and their variations are presented in Figure 3. The final, fabricated laminates are shown in Figure 4. The motivation behind these different "scenarios" of colored and patterned coated glass of the tested PV laminates was to allow a sufficient comparison of the impact of different colors (for the same pattern) and of different patterns (for the same color). It should be noted that all compared patterned samples, have the same shading fraction, equal to $50 \%$, independent of the pattern design (linear, dotted or squared). We then performed

$$
\begin{aligned}
& M 2=R_{v}+T_{v}^{2} \cdot R_{p}+T_{v}^{2} \cdot T_{p}^{2} R_{a} \\
& M 1=R_{v}+T_{v}^{2} \cdot R_{p}+T_{v}^{2} \cdot T_{p}^{2} R_{e}
\end{aligned} \square \quad \begin{aligned}
& T_{p}=\frac{1}{T_{v}} \sqrt{\frac{M 1-M 2}{R_{e}-R_{a}}} \\
& R_{p}=\frac{1}{T_{v}^{2}} \cdot\left(M 2-R_{v}-T_{v}^{2} \cdot T_{p}^{2} R_{a}\right)
\end{aligned}
$$



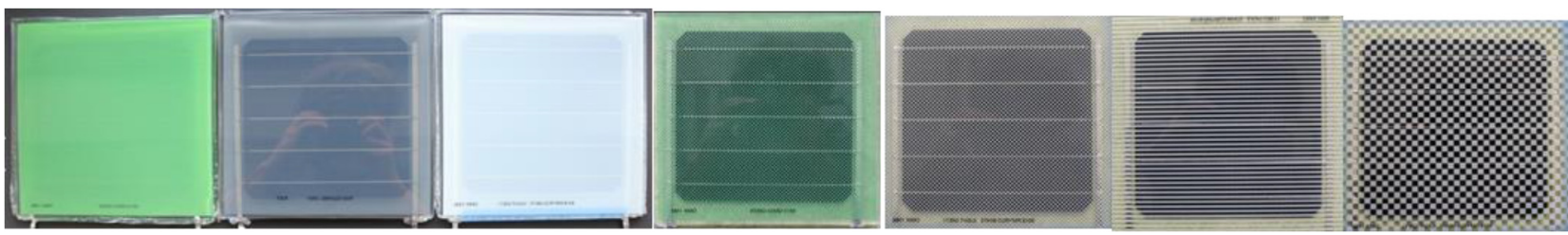

Fig. 4. The fabricated 1-cell modules/laminates with front glass coated with different colors (from left to right: grass green, squirrel grey, papyrus white, grass green dotted, papyrus white dotted, papyrus white linear and papyrus white squared).

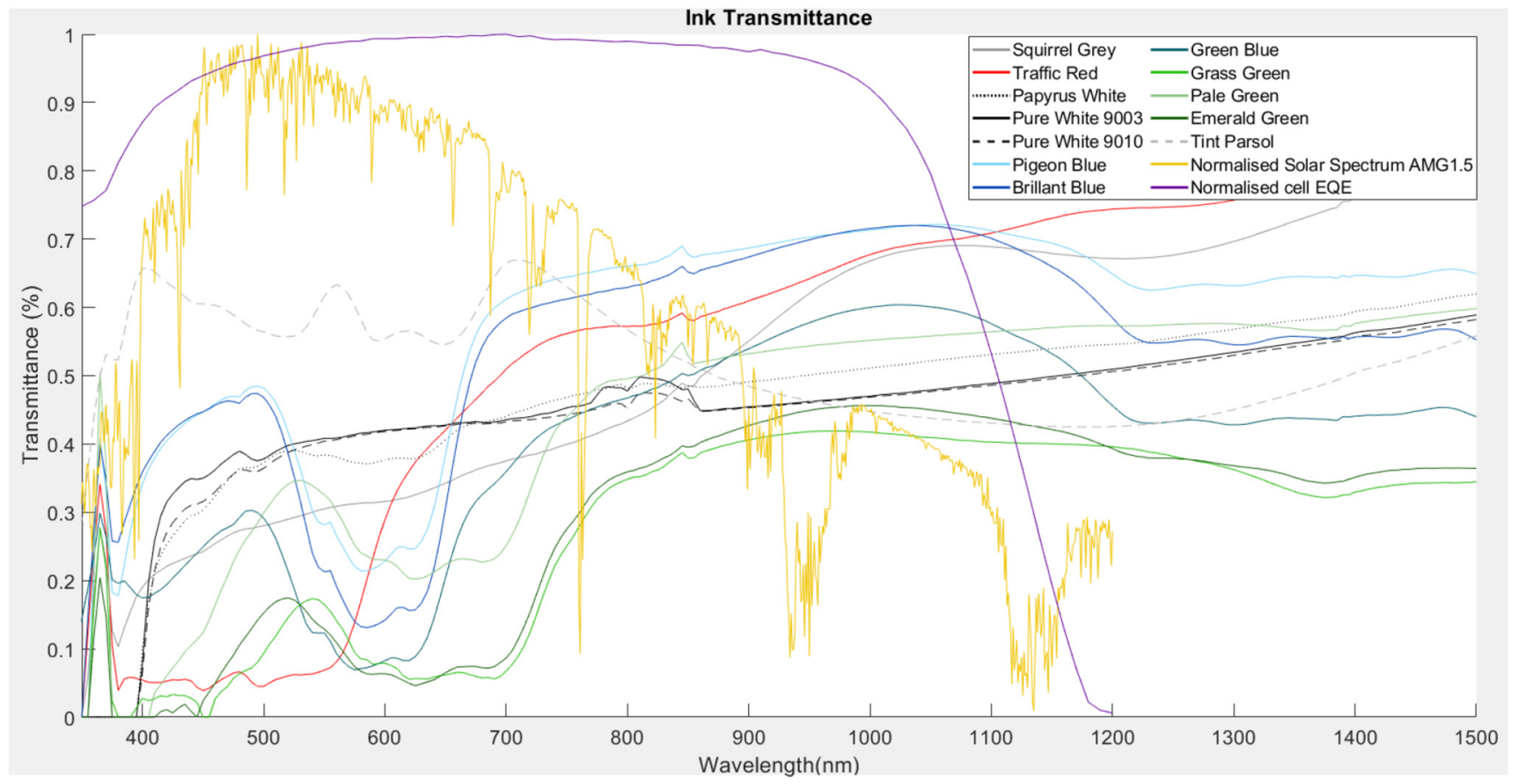

Fig. 5. Transmission spectra measured and compared for the 12 different colors of Set 2.

electrical $(I-V)$ characterization of the PV laminates, both at STC and at different intensity (irradiance) levels $\left(1000 \mathrm{~W} / \mathrm{m}^{2}\right.$, $600 \mathrm{~W} / \mathrm{m}^{2}, 400 \mathrm{~W} / \mathrm{m}^{2}$ and $200 \mathrm{~W} / \mathrm{m}^{2}$ ) and at different angles of incidence $\left(0^{\circ}, 20^{\circ}, 45^{\circ}\right.$ and $\left.70^{\circ}\right)$, to quantify and understand better the impact of the different coated glasses on the main electrical parameters of the PV laminates. The $I$ - $V$ characterization measurements were performed using a Xenon Single Long-Pulse solar simulator (Spire/EternalSun 5100SLP Blue), class $\mathrm{A}+\mathrm{A}+\mathrm{A}+$ (per IEC 60904-9 ed. 3) for spectrum, spatial uniformity, and temporal stability. The $I-V$ characterization measurements with angle variations were performed using a Xenon $10 \mathrm{~ms}$ Pulse solar simulator (Pasan Measurement Systems HighLight 3), class $\mathrm{A}+\mathrm{A}+\mathrm{A}+$, in a $8 \mathrm{~m}$ long tunnel with half-collimated light, and using an angular measuring support at the same time.

\section{Results and discussion}

\subsection{Optical performance}

In overall, the performed measurements determined the transmittance, reflectance and absorbance values of each of the 25 different colour coatings, for a PV-relevant spectrum, i.e. from $300 \mathrm{~nm}$ to $1500 \mathrm{~nm}$, with intervals (steps) of $5 \mathrm{~nm}$. On the basis of the transmittance on the Solar Spectrum and the EQE characteristics of a solar cell, we can assess the short-circuit current and so the "efficiency" of each coating compared to a non-coated PV module, for potential use for colored EPoG and BIPV applications. Indicatively, in Figure 5 we present the resulting measured spectra for the light transmission measurements of Set 2 ("newest" printing recipe for the colored BIPV glazing). In this case, the best performing colors for the overall spectrum are blue shades RAL5014 and RAL5007 with respectively $60 \%$ and $57 \%$ of efficiency compared to a non-coated PV Module, followed by the RAL3020 ("Traffic Red") with 51\%. Then, the grey and white shades (RAL 7000, RAL9018, RAL9010, RAL9003) ranging between 43 and $48 \%$. Finally, the worst performers are the green shades RAL6021, RAL5001, RAL6001 and RAL6010, ranging respectively from $43 \%$ to $28 \%$ of efficiency (see also Tab. 1).

With a similar analysis-observation of the resulting spectra for Set 1 (i.e. the first color coating recipe), we concluded that the best (by far) performing color/coating (in the overall spectrum) for that recipe, is the RAL9018 ("Papyrus White") with about 70\%. The RAL5014 ("Pigeon Blue") and the RAL3020 ("Traffic red") also 
Table 1. Overview of the obtained average efficiencies (compared to non-coated PV glass) from the measured transmittance for the 12 colored coatings of Set 1 and 2.

\begin{tabular}{lll}
\hline Color & $\begin{array}{l}\text { Average efficiency* } \\
\text { for Set 1 }\end{array}$ & $\begin{array}{l}\text { Average efficiency* } \\
\text { for Set 2 }\end{array}$ \\
\hline Squirrel Grey RAL7000 & $47 \%$ & $48 \%$ \\
Traffic Red RAL3020 & $57 \%$ & $51 \%$ \\
Papyrus White RAL9018 & $70 \%$ & $47 \%$ \\
Pure/Signal White RAL9003 & $49 \%$ & $43 \%$ \\
Pure White RAL9010 & $47 \%$ & $45 \%$ \\
Pigeon Blue RAL5014 & $57 \%$ & $60 \%$ \\
Brilliant Blue RAL5007 & $49 \%$ & $57 \%$ \\
Green Blue RAL5001 & $47 \%$ & $43 \%$ \\
Grass Green RAL6010 & $29 \%$ & $28 \%$ \\
Pale Green RAL6021 & $30 \%$ & $43 \%$ \\
Emerald Green RAL6001 & $28 \%$ & $29 \%$ \\
Tint Parsol & $\mathrm{n} / \mathrm{a}$ & $42 \%$ \\
\hline
\end{tabular}

${ }^{*}$ relative values, compared to non-coated PV glass transmittance).

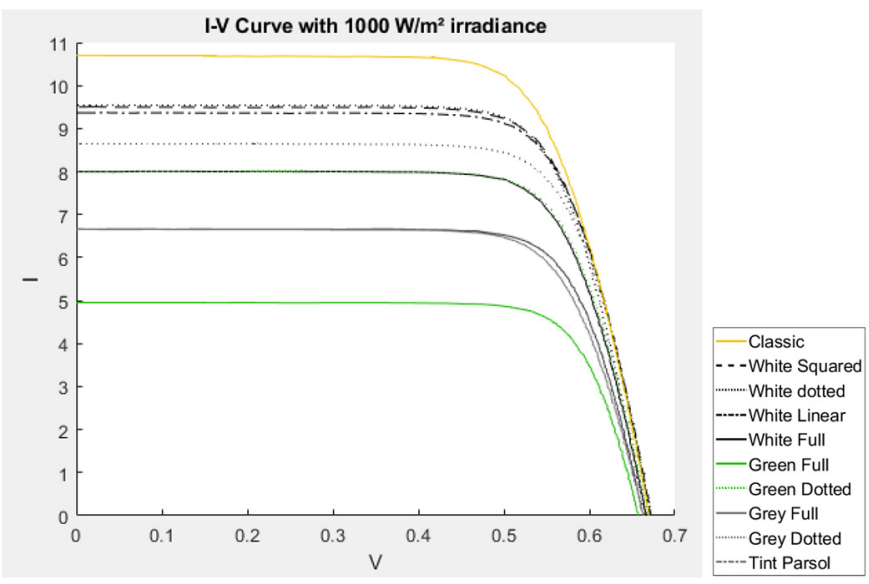

Fig. 6. $I-V$ characteristics measured at $1000 \mathrm{~W} / \mathrm{m}^{2}$, for the different color/pattern coated PV laminates of the study.

performed relatively well with about $57 \%$ of efficiency for both. Then, the white shades RAL9003, RAL9010 and RAL7000 ("Signal White", "Pure White" and "Squirrel Grey") and certain shades in the green family - such as the RAL6021 ("Pale Green"), RAL6010 or RAL6001-show an inferior performance with an efficiency in a range of $28 \%-49 \%$.

\subsection{Electrical performance (light intensity dependence)}

Figure 6 depicts how all measured $I-V$ characteristics are compared for the different color/pattern coated PV laminates, for the $1000 \mathrm{~W} / \mathrm{m}^{2}$ irradiance level (STC). Indicatively, Table 2 provides an overview of the main electrical parameters measured for the $10 \mathrm{PV}$ laminates, for the case of $600 \mathrm{~W} / \mathrm{m}^{2}$ irradiance level.
In general, we observe that, in terms of colored coating type, the RAL9018 is consistently the best performer (after the reference) among the uniform (100\% coating coverage) color coatings, in terms of power and current output, for the same irradiance level. For instance, at $1000 \mathrm{~W} / \mathrm{m}^{2}$, the PV laminate with uniform RAL9018 has a $P_{\max } 22 \%$ higher compared to RAL7000 and $53 \%$ higher compared to RAL6010 and $23 \%$ higher compared to the ParSol. On the other hand, when comparing the different patterned coatings (for the same color, RAL9018 in this study), we conclude that the linear pattern on the PV laminates results in the best performance $\left(P_{\max }\right.$ and output current), while - as expected - the uniform $100 \%$ coating covered PV laminates have the lowest performance, compared to the patterned ones. In particular, e.g. at $1000 \mathrm{~W} / \mathrm{m}^{2}$, the linear pattern of RAL9018 gives a $P_{\max } 1.5 \%$ higher compared to the squared pattern, $2 \%$ higher compared to the dotted pattern and $20.4 \%$ higher compared to the uniform $(100 \%)$ one. However, it can be affirmated that using RAL9018 linear pattern offers the best performance compared to others patterns, considering that it can be associated with the printed vs "clear" area surface ratio. Also, considering the non-coated PV laminate as a reference, we observe that the the patterned coating solutions result in lower losses $(-10.5 \%)$ in $P_{\max }$ compared to the grey tint or the uniform RAL6010, which present $P_{\max }$ losses of $-45 \%$ and $-66.4 \%$ respectively. Similar findings amd conclusions are observed through the complete set of electrical measurements ( $I-V$ and $P-V$ characteristics) in all aforementioned irradiance levels.

\subsection{Electrical performance (angle-of-incidence dependence)}

In Table 3 we provide an overview of the main electrical parameters measured for the $10 \mathrm{PV}$ laminates at the four different angles-of-incidence (AoI), at $1000 \mathrm{~W} / \mathrm{m}^{2}$ 
Table 2. Electrical parameters for $10 \mathrm{PV}$ laminates with different color/pattern coatings after IV characterization at $600 \mathrm{~W} / \mathrm{m}^{2}$.

\begin{tabular}{lllllll}
\hline & $P_{\max }(\mathrm{W})$ & $I_{\mathrm{sc}}(\mathrm{A})$ & $V_{\mathrm{oc}}(\mathrm{V})$ & $I_{\mathrm{mpp}}(\mathrm{A})$ & $V_{\mathrm{mpp}}(\mathrm{V})$ & $F F(\%)$ \\
\hline Reference & 4.55 & 9.83 & 0.669 & 8.95 & 0.508 & 69.1 \\
RAL9018 Linear & 4.07 & 8.33 & 0.667 & 7.8 & 0.520 & 73.2 \\
RAL9018 Squared & 4.01 & 8.23 & 0.668 & 7.64 & 0.525 & 73.0 \\
RAL9018 Dotted & 3.99 & 8.26 & 0.662 & 7.66 & 0.521 & 73.0 \\
RAL9018 100\% & 3.24 & 6.53 & 0.662 & 6.11 & 0.530 & 74.9 \\
RAL7000 Dotted & 3.54 & 7.18 & 0.665 & 6.69 & 0.529 & 74.2 \\
RAL7000 100\% & 2.51 & 5.01 & 0.654 & 4.69 & 0.534 & 76.5 \\
RAL6010 Dotted & 3.24 & 6.54 & 0.662 & 6.14 & 0.529 & 74.8 \\
RAL6010 100\% & 1.53 & 3.44 & 0.646 & 2.82 & 0.545 & 69.1 \\
Grey Tint ParSol & 2.50 & 5.04 & 0.654 & 4.74 & 0.527 & 75.8 \\
\hline
\end{tabular}

irradiance level; while Figure 7 shows the correlation of $P_{\max }$ and the evolution of $P_{\max }$ losses as a function of the angle-of-incidence, for all different tested PV laminates, at the $1000 \mathrm{~W} / \mathrm{m}^{2}$ irradiance level. In overall, we observe that, independently of the color coating (reference, uniform or pattern color), the performance (in terms of $P_{\max }$, in this case) loss rate clearly increases for angles-of-incidence beyond $45^{\circ}$. This observation is in general agreement with earlier experimental and modelling studies on the AoI-dependent performance losses of PV modules [7-9]; besides - for the patterned coatings, in the specific study higher power output losses can be further explained by a higher effective shading fraction (and thus transmittance losses) for AoI beyond $45^{\circ}$.

\section{Summary - concluding remarks and future work}

We presented an experimental comparative study on the optical and electrical performance of multiple color coated and patterned BIPV glazing solutions, towards their upscaling and commercialization. In this context, we performed optical transmission measurements and light intensity-/angle-depent IV characterization on 25 different colored glass samples and 10 different colored/patterned glass PV laminates respectively.

The outcomes provide preliminary yet valuable insights into the optical-electrical performance of the investigated colored BIPV glazing, as well as a first identification of BIPV industry-relevant colors and patterns with the best potential "compromise" between aesthetics and performance, for future energy positive glazing applications. In brief, we may summarize the following main "takeaways":

- Color coatings for glass in grey, white and blue shades, as the best performers in terms of measured transmittance, demonstrate the best efficiency and best (optical) performance vs aesthetics compromise, while the green shades family are performing the worst.

- The electrical $(I-V)$ characterization study, at different irradiance levels, confirmed the observations from the transmittance measurements on certain tested colors; and, in addition, it indicated that different patterns (squares, dots, lines) present similar overall performance for the same color, when compared between them.

- The angle-dependent $I-V$ characterization indicated that power losses evolve at a higher rate for angle-of-incidence beyond $45^{\circ}$, while the performance loss rate varies slightly in function of the angle-of-incidence and the tested pattern/color coating.

It must be highlighted, of course, that the above observations and outcomes, on the best-performing color shades for BIPV glazing, should not be taken as "absolute" conclusions, but rather applicable for the specific type and technology of color (ceramic, digitally printed) coated BIPV glazing. Certain parameters, such as the thickness of the color layer or the charge of the color pigments, may have also significant impact on the overall optical and electrical performance of such BIPV coloring approaches.

As steps forward in future work, our research will aim to yield additional insights and understanding into: (i) the longterm reliability and qualification of such coated BIPV glazing solutions, under accelerated (combined and individual) stress tests, (ii) the impact of the coating's disposition in the BIPV modules layout (air-side vs encapsulant-side) on both the performance and the reliability of future colored EPoG modules, (iii) the shading/mismatch effects, supported by optical modelling/simulations for different colors, shapes, patterns and textures.

The authors acknowledge the valuable contribution of all partners involved in the H2020 BE-SMART project. This project has received funding from the European Union's Horizon 2020 research and innovation programme under grant agreement 818009 . Contribution in this work was also supported in the context of INES.2S, funded from the French State under the programme "Investissements d'Avenir", with reference ANR-10-IEED-0014-01.

\section{Author contribution statement}

B. Riedel conducted the entirety of the experimental work (set-up, measurements and analysis), i.e. in relation to both the optical and the electrical characterization study, thus contributing in the images/plots production and discussion parts mainly of Section 3 . 


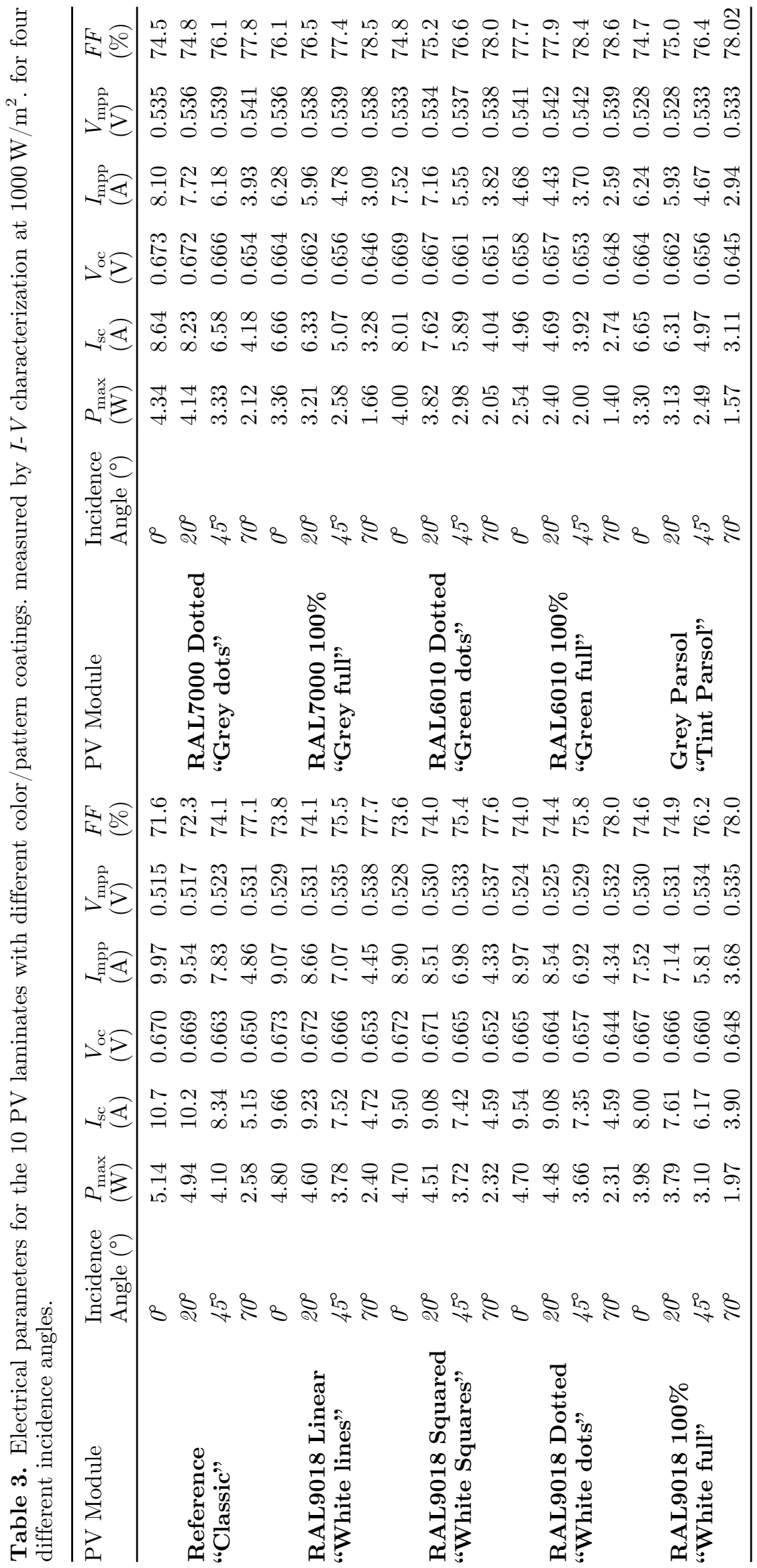



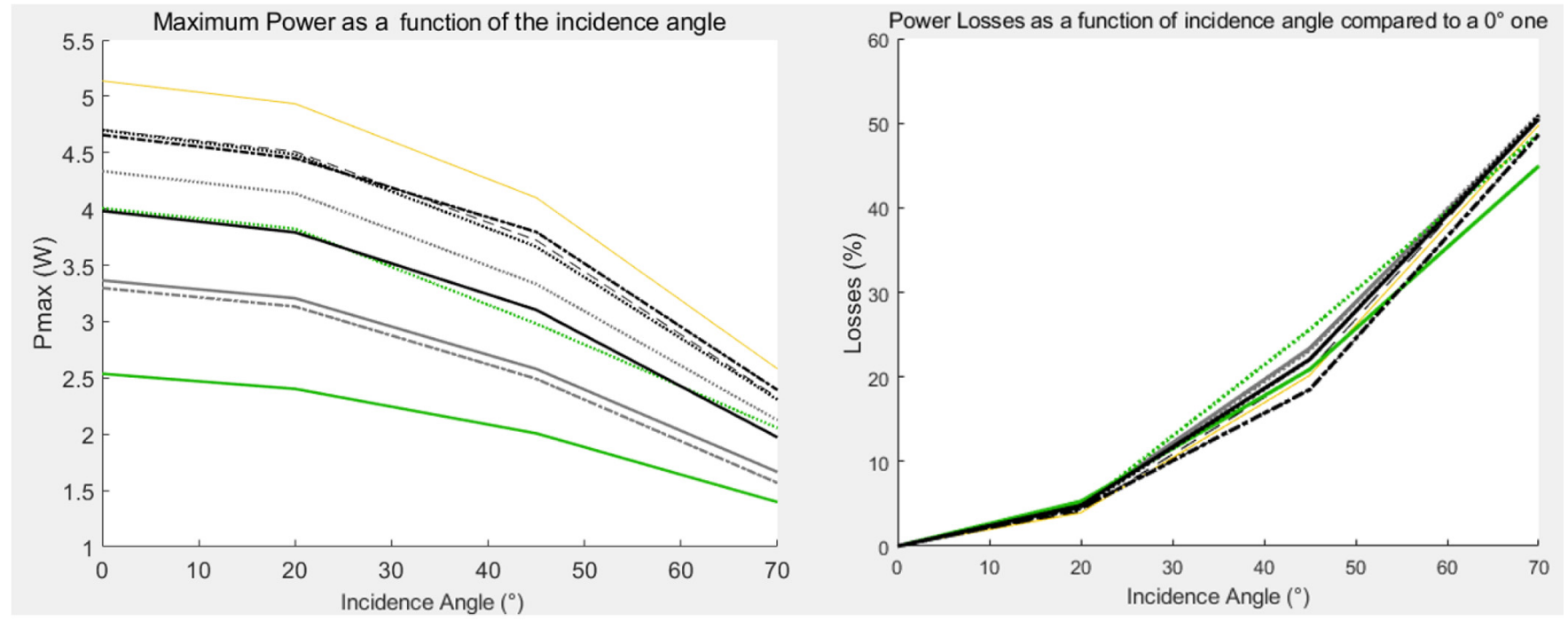

\begin{tabular}{|c|c|c|c|c|}
\hline $\begin{array}{l}- \text { Classic } \\
-\quad-\text { White Squared }\end{array}$ & ......... White dotted & $\begin{array}{l}\text { White Full } \\
\text { Green Full }\end{array}$ & $\begin{array}{c}\text {............ Green Dotted } \\
\text { Grey Full }\end{array}$ & $\begin{array}{l}\text {............ Grey Dotted } \\
-=-=- \text { Tint Parsol }\end{array}$ \\
\hline
\end{tabular}

Fig. 7. Measured $P_{\max }$ absolute values and $P_{\max }$ losses as a function of the angle-of-incidence, at $1000 \mathrm{~W} / \mathrm{m}^{2}$, for the different color/ pattern coated PV laminates of the study.

P. Messaoudi provided the technical support and supervision in the experimental work, particularly in relation to the fabrication and the bill-of-materials (BOM) definition of the tested minimodules. Y.B. Assoa and $\boldsymbol{P}$. Thony contributed on the theoretical groundwork of this study, the definition of the objectives and the overall scientific supervision and review of the study. R. Hammoud provided (on behalf of Saint-Gobain) all the different colored and patterned glass samples characterized or used in this study. He also provided insights into the colorization/coating method and recipes. L-E. Perret-Aebi contributed in this study, from her function as coordinator of BESMART project, contributing to the overall scientific supervision and review of the study and parts in Sections 1 and 4. J.A. Tsanakas contributed with writing and editing mostly of sections 1, 2 and 4, the review and correspondence for the final paper, the revisions and (at the time of writing of this paper) the overall coordination and workplan definition of the project team, together with Y.B. Assoa, on behalf of CEA.

\section{References}

1. IEA PVPS Task 15 Subtask C - International framework for BIPV specifications, "Analysis of requirements, specifications and regulation of BIPV", Report IEA-PVPS T15-08: 2019
2. BIPV Technologies and Markets 2017-2026, n-tech Research Report, 2017

3. EU H2020 BE-SMART Project: [https://www.besmartproject. $\mathrm{eu} /]$

4. N. Sánchez-Pantoja, R. Vidal, M. Carmen Pastor, Aesthetic perception of photovoltaic integration within new proposals for ecological architecture, Sustain. Cit. Soc. 38, 203 (2018)

5. M. Pelle, E. Lucchi, L. Maturi, A. Astigarraga, F. Causone, Coloured BIPV technologies: methodological and experimental assessment for architecturally sensitive areas, Energies 13, 4506 (2020)

6. G. Eder et al., Coloured BIPV: Market, Research and Development. Report IEA-PVPS T15-07: 2019

7. D.L. King, J.A. Kratochvil, W.E. Boyson, Measuring solar spectral and angle-ofincidence effects on photovoltaic modules and solar irradiance sensors, in IEEE Photovoltaic Specialists Conference, Anaheim, California, 1997

8. D.L. King, E.E. Boyson, J.A. Kratochvil, Photovoltaic Array Performance Model (Sandia National Laboratories, Albuquerque, NM, 2004), SAND2004-3535

9. Sandia IAM Model. Available at: https://pvpmc.sandia.gov/ modeling-steps /1-weather-design-inputs/shading-soiling-andreflection-losses/incident-angle-reflection-losses/sandia-model/

Cite this article as: Benjamin Riedel, Paul Messaoudi, Ya Brigitte Assoa, Philippe Thony, Rayan Hammoud, LaureEmmanuelle Perret-Aebi, John A. Tsanakas, Color coated glazing for next generation BIPV: performance vs aesthetics, EPJ Photovoltaics 12, 11 (2021) 